\title{
Watershed subarachnoid hemorrhage after middle cerebral artery rescue stenting in patients with acute ischemic stroke
}

\author{
Francesco Diana ${ }^{1}$ (D) $\cdot$ Maria Di Gregorio ${ }^{2} \cdot$ Giulia Frauenfelder $^{1} \cdot$ Renato Saponiero $^{1} \cdot$ Daniele Giuseppe Romano $^{1}$
}

Received: 26 January 2021 / Accepted: 15 March 2021 / Published online: 24 March 2021

(C) The Author(s) 2021

\begin{abstract}
Cortical subarachnoid hemorrhage is an infrequent subtype of non-aneurysmal subarachnoid hemorrhage, rarely reported in watershed territories (wSAH) after carotid stenting. It has never been reported after treatment of middle cerebral artery stenosis (MCAS) that is increasingly used in selected patients, as rescue treatment of failed mechanical thrombectomy, due to recent advancements in endovascular interventions. We present a series of patients with MCAS that developed a wSAH after stenting.
\end{abstract}

Keywords Intracranial stenosis $\cdot$ MCAS $\cdot$ Intracranial stenting $\cdot$ Rescue stenting $\cdot$ SAH $\cdot$ ICH $\cdot$ Hyperperfusion syndrome $\cdot$ HPS

$\begin{array}{ll}\text { Abbreviations } \\ \text { ACA } & \begin{array}{l}\text { Anterior cerebral artery } \\ \text { Acute ischemic stroke } \\ \text { AIS }\end{array} \\ \text { ASITN/SIR } & \begin{array}{l}\text { American Society of Intervention } \\ \text { and Therapeutic Neuroradiology/Society } \\ \text { of Interventional Radiology }\end{array} \\ \text { BBB } & \begin{array}{l}\text { Blood-brain barrier } \\ \text { Intracranial arterial stenosis }\end{array} \\ \text { IAS } & \text { Leptomeningeal artery } \\ \text { LMA } & \text { Middle cerebral artery stenosis } \\ \text { MCAS } & \text { Thrombolysis in Cerebral Infarction } \\ \text { TICI } & \text { Watershed subarachnoid hemorrhage } \\ \text { wSAH } & \end{array}$

al cisterns, ventricles, Sylvian fissure or interhemispheric fissure, and so on. Cortical SAH of watershed territories (wSAH) has been described after carotid endarterectomy or stenting rarely [1], and has never been reported after treatment of IAS.

Recent studies demonstrated that rescue stenting (RS) after failed mechanical thrombectomy increases the rate of good clinical outcome and does not affect the intracranial hemorrhage or mortality rates $[2,3]$. Hence, stenting of IAS is increasingly used in selected patients and the knowledge of its effects is mandatory. We present a series of cases developing a wSAH after intracranial stenting of MCAS.

\section{Cases}

\section{Introduction}

Cortical subarachnoid hemorrhage (cSAH) is an infrequent subtype of non-aneurysmal SAH localized in one or a small number of brain cortex sulcus, without spreading into the bas-

Francesco Diana

francesco.diana.md@gmail.com

1 Department of Neuroradiology, A.O.U. San Giovanni di Dio e Ruggi d'Aragona, University of Salerno, Salerno, Italy

2 Department of Neurology, A.O.U. San Giovanni di Dio e Ruggi d'Aragona, University of Salerno, Salerno, Italy

Between January and November 2020 we performed 10 stenting in patients with AIS due to intracranial atherosclerotic disease: four patients with vertebra-basilar stenosis and six patients with MCAS. Four patients, 2 female and 2 male with a mean age of 70 years (range, 53-85 years), developed a wSAH after treatment, all of them with MCAS. We reviewed our institutional database and reported patients' baseline characteristics in Table 1.

All patients had an ASPECT score $>6$ and presented with a mean NIHSS of 9 (range, 5-16). At the CT-angiography (CTA), the MCA was occluded 2 patients, and sub-occluded in the others. MCA stenting was a rescue therapy in three cases and the first-line therapy in the other one (case 3). 


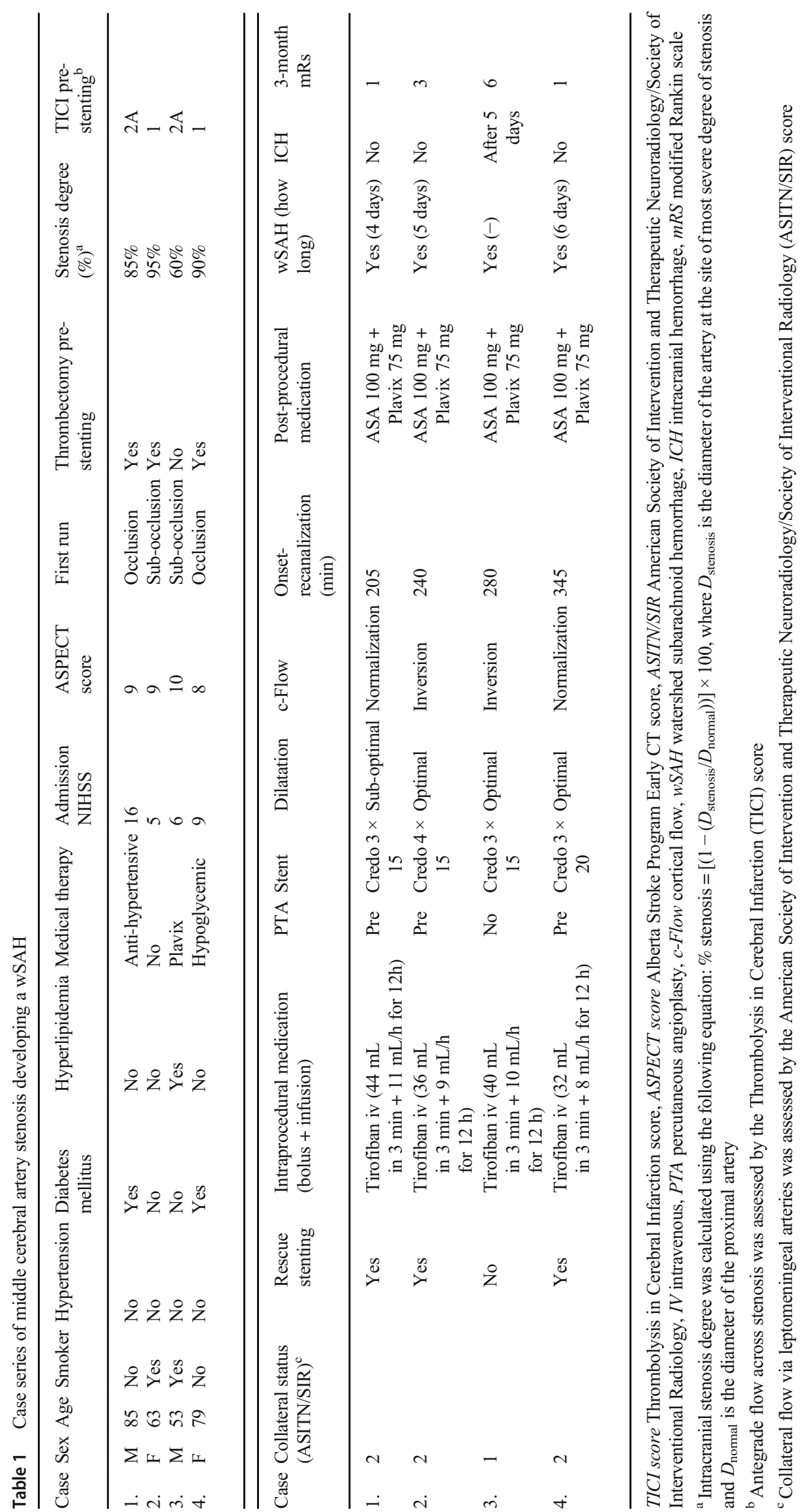



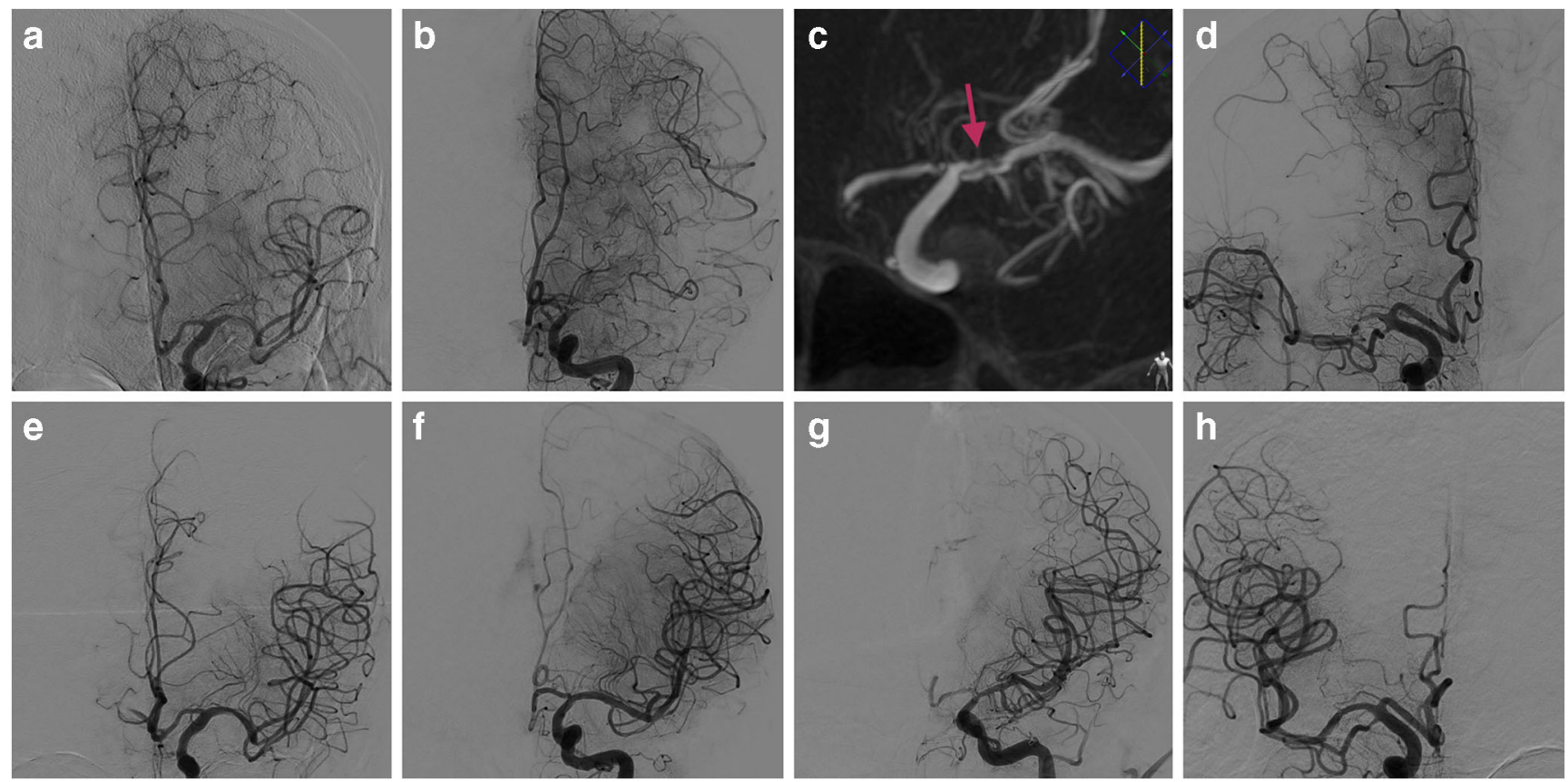

Fig. 1 Cases 1/2/3/4. a, b, and $\mathbf{d}$ Angiographic aspect of middle cerebral artery stenosis before rescue stenting. $\mathbf{c}$ Cone-beam CT angiography showing a dissected plaque (red arrow). $\mathbf{e}-\mathbf{h}$ Angiographic control after stenting

Patient 3 was admitted with fluctuating neurological deficits caused by a dissected plaque (Fig. 1).

Among all patients, the mean MCAS was $82 \%$ (range, 60 95\%).

Considering the antegrade flow, contrast failed to opacify distal cerebral territories (TICI 1 ) in two patients, while perfused less than $2 / 3$ of the entire vascular bed (TICI $2 \mathrm{~A}$ ) in the other two. The leptomeningeal collateral flow from the anterior cerebral artery (ACA) to the ischemic site was slow and peripheral (score 1) in one patient, fast and peripheral (score 2) in three of them.

All procedures were performed without intraprocedural heparin. We administered to all patients a loading dose and continuous infusion of Tirofiban. Then, we deployed a selfexpanding stent (Credo, Acandis GmbH Pforzheim, Germany) at the level of the MCAS, with or without angioplasty, obtaining a complete dilatation within $6 \mathrm{~h}$ from the symptom onset (Table 1). At the control angiogram, collateral flow from the ACA to MCA territories, which had been present at the time of occlusion, disappeared, but leptomeningeal arteries (LMAs) of watershed territories were still dilated. Moreover, we observed a prolonged contrast staining within these LMAs in the late phase of the angiogram, with competing flow between the ACA and the MCA in two cases, and inverted flow from the MCA to ACA territories in the other two (Fig. 2a-d).

wSAH immediately appeared at the post-procedural CT control in all patients (Fig. 2), and disappeared after a mean time of 5 days without neurological consequences. However, clinical course of patient 3 was complicated by a deathly left basal ganglia hemorrhage, 5 days after treatment; until then he recovered without focal neurological deficits and brain ischemic lesions.

\section{Discussion}

Contrast enhancement hyper-attenuation $(\mathrm{CEH})$ is defined as a benign finding, mimicking a $\mathrm{SAH}$, that shows progressive resolution within $24 \mathrm{~h}$, in which the measured Hounsfield units are less than 70 [4], and has been described after different neurovascular procedures [4-7]. It may be induced by several factors, such as high amounts of contrast medium and transient hemodynamic changes during treatment [4]. Regarding the pathogenic mechanism, Yoon et al. [8] postulated that $\mathrm{CEH}$ is caused by reversible injuries of the blood-brain barrier (BBB), involving the inter-endothelial tight junctions. Instead, SAH has been described after mechanical thrombectomies due to vessel perforation or mechanic destruction of the endothelial integrity and is localized within the basal cisterns. Only rare cases reported a SAH in distal territories, but associated with focal cortical ischemia [9]. Our finding, however, is different from that reported in literature: it was an extravasation of blood within the pial surface and the adjacent subarachnoid space, not associated with cortical ischemia, that resolved after a mean time of 5 days.

Among all patients with AIS due to intracranial stenosis treated between January and November 2020, four of them with MCAS developed the wSAH after treatment, while two with MCAS and four with VBS did not. We were not able to 

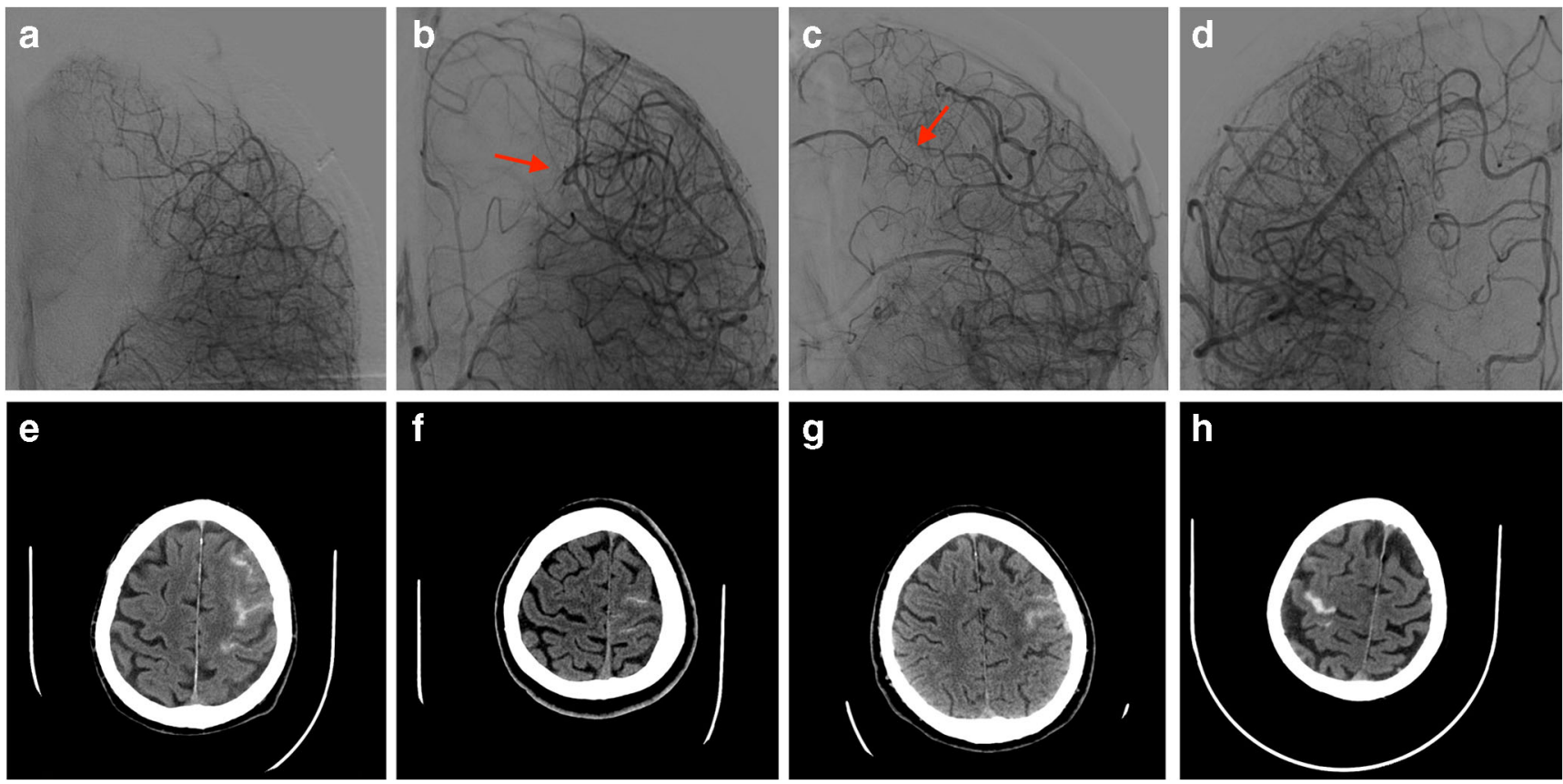

Fig. 2 Cases 1/2/3/4. a-d Capillary phase of the angiographic control after stenting showing the hemodynamic overload of leptomeningeal arteries and the inversion of flow in two patients (red arrow). $\mathbf{e}-\mathbf{h}$ CT control showing watershed subarachnoid hemorrhage

compare the two groups, due to the limited number of cases. Although, we decided to describe this phenomenon and to analyze patients' characteristics potentially related to it.

wSAH reported in our cases might have biological and hemodynamic causes, related with the pathophysiology of the MCAS. On one side, the MCAS leads a downstream hemodynamic stress that increases the compensatory capacity of the LMAs [10, 11], by reducing their vasoreactivity [12]. On the other side, the brain perfusion alteration induced by the MCAS determine a subclinical ischemic condition that increases the permeability of the BBB [13]. Hence, the sudden restoration of distal flow, that we want to achieve with MCAS angioplasty and stenting, could determine an overload of distal vessels with an altered BBB and no longer able to tolerate normal hydraulic pressures, resulting in blood extravasation in the subarachnoid space. Before treatment, we found dilated LMAs in watershed ACA/MCA territories with collateral flow from the ACA to MCA territories in all patients of this series; it may suggest that these patients are prone to develop wSAH after treatment.

Three patients of this series presented with high MCAS degree and good collateral status. In symptomatic patients, these elements are strictly linked: the trans-stenosis pressure gradient $(\mathrm{PG})$ is an independent predictor of good leptomeningeal collateral status [11], and both are signs of brain hemodynamic compromise [14]. In our opinion, MCAS degree and collateral status might be predictive factors of wSAH.

The AIS of patient 3 was caused by a dissected plaque that determined hemodynamic failure to the distal vascular territories. In this case the MCAS degree and the leptomeningeal collaterals were not directly linked; LMAs were dilated, as in cases of chronic occlusion, despite the stenosis degree was low. Although the pathophysiology of this AIS was different, there was still an association between LMA hypertrophy and the wSAH.

Blood pressure values can affect the development of the collateral circulation in patients with intracranial stenosis. Thus, an inverse correlation between the blood pressure and the risk of wSAH might exist. Hypertension impairs the angiogenic process that leads to the development of pial collaterals [15] and increases the myogenic tone of LMAs. In such patients, LMAs are high-resistance vessels with a reduced baseline diameter and a higher myogenetic tone that increases their breaking strength, while in normotensive patients they are larger and do not have a myogenetic response to the blood pressure variations [16]. All patients of our series were admitted with history of normal blood pressure, endorsing this theory.

All patients of this series were anti-aggregated with a loading dose and a continuous infusion of Tirofiban. In our experience, we have never seen the wSAH in patients with embolic AIS; hence, we could assume that the antiaggregation and the wSAH might be related. We can hypothesize that the anti-aggregation might increase the amount of blood extravasation within the subarachnoid space; however, we do not think that it could be the cause of this phenomenon. To our knowledge, the wSAH has never been reported in patients anti-aggregated with Tirofiban for other cerebrovascular diseases. 
wSAH might precede a hyper-perfusion syndrome, as observed in patient 3 . In this patient, the MCAS was located before the origin of the lenticulostriate arteries. We could speculate that biological and hemodynamic alterations, caused by the intracranial stenosis, involved both the LMAs and the lenticulostriate arteries. However, since the main limitation of this study is the number of patients, the clinical relevance of the wSAH needs further studies.

\section{Conclusion}

wSAH may present after MCAS stenting. Pathophysiology of the wSAH can be linked with biological and hemodynamic alterations of cerebral autoregulation mechanism. wSAH might be predicted by some anamnestic and anatomical factors and could be associated with the hyper-perfusion syndrome. Further studies are needed to clarify its clinical relevance.

\section{Acknowledgements None.}

Author contribution Conception and design of the work: FD. Data acquisition: FD, MDG. Data analysis and interpretation: FD, GF. Drafting the work: FD and DGR. Critical revision: DGR, RS. Final approval: all authors.

Funding Open access funding provided by Università degli Studi di Salerno within the CRUI-CARE Agreement.

\section{Declarations}

Ethical approval All procedures performed in the studies involving human participants were in accordance with the ethical standards of the institutional and/or national research committee and with the 1964 Helsinki Declaration and its later amendments or comparable ethical standards.

Informed consent Informed consent was obtained from all individual participants included in the study.

Conflict of interest The authors declare that they have no conflict of interest.

Open Access This article is licensed under a Creative Commons Attribution 4.0 International License, which permits use, sharing, adaptation, distribution and reproduction in any medium or format, as long as you give appropriate credit to the original author(s) and the source, provide a link to the Creative Commons licence, and indicate if changes were made. The images or other third party material in this article are included in the article's Creative Commons licence, unless indicated otherwise in a credit line to the material. If material is not included in the article's Creative Commons licence and your intended use is not permitted by statutory regulation or exceeds the permitted use, you will need to obtain permission directly from the copyright holder. To view a copy of this licence, visit http://creativecommons.org/licenses/by/4.0/.

\section{References}

1. Larrosa D, Ramon C, Benavente L, Calleja S (2016, 2016) Convexity subarachnoid haemorrhage secondary to internal carotid stenosis: an indication for revascularisation. BMJ Case Rep. https:// doi.org/10.1136/bcr-2016-214661

2. Maingard J, Phan K, Lamanna A, Kok HK, Barras CD, Russell J, Hirsch JA, Chandra RV, Thijs V, Brooks M, Asadi H (2019) Rescue intracranial stenting after failed mechanical thrombectomy for acute ischemic stroke: a systematic review and meta-analysis. World Neurosurg 132:e235-e245. https://doi.org/10.1016/j.wneu. 2019.08.192

3. Chang Y, Kim BM, Bang OY, Baek JH, Heo JH, Nam HS, Kim YD, Yoo J, Kim DJ, Jeon P, Baik SK, Suh SH, Lee KY, Kwak HS, Roh HG, Lee YJ, Kim SH, Ryu CW, Ihn YK, Kim B, Jeon HJ, Kim JW, Byun JS, Suh S, Park JJ, Lee WJ, Roh J, Shin BS, Kim JM (2018) Rescue stenting for failed mechanical thrombectomy in acute ischemic stroke: a multicenter experience. Stroke 49:958964. https://doi.org/10.1161/STROKEAHA.117.020072

4. Brisman JL, Jilani M, McKinney JS (2008) Contrast enhancement hyperdensity after endovascular coiling of intracranial aneurysms. AJNR Am J Neuroradiol 29:588-593. https://doi.org/10.3174/ajnr. A0844

5. Nakano S, Iseda T, Kawano H, Yoneyama T, Ikeda T, Wakisaka S (2001) Parenchymal hyperdensity on computed tomography after intra-arterial reperfusion therapy for acute middle cerebral artery occlusion: incidence and clinical significance. Stroke 32:20422048. https://doi.org/10.1161/hs0901.095602

6. Shah PR, Yohendran J, Parker GD, McCluskey PJ (2013) Contrastinduced transient cortical blindness. Clin Exp Optom 96:333-335. https://doi.org/10.1111/j.1444-0938.2012.00786.x

7. Ozturk A, Saatci I, Pamuk AG, Erdogan C, Akmangit I, Geyik S, Cekirge HS (2006) Focal increased cortical density in immediate postembolization CT scans of patients with intracranial aneurysms. AJNR Am J Neuroradiol 27:1866-1875

8. Yoon W, Seo JJ, Kim JK, Cho KH, Park JG, Kang HK (2004) Contrast enhancement and contrast extravasation on computed tomography after intra-arterial thrombolysis in patients with acute ischemic stroke. Stroke 35:876-881. https://doi.org/10.1161/01. STR.0000120726.69501.74

9. Nikoubashman O, Reich A, Pjontek R, Jungbluth M, Wiesmann M (2014) Postinterventional subarachnoid haemorrhage after endovascular stroke treatment with stent retrievers. Neuroradiology 56:1087-1096. https://doi.org/10.1007/s00234014-1424-1

10. Liu X, Dornbos D, Pu Y et al (2017) Collateral circulation alters downstream hemodynamic stress caused by intracranial atherosclerotic stenosis. Neurol Res 39:498-503. https://doi.org/10.1080/ 01616412.2017.1315483

11. Leng X, Lan L, Ip HL, Fan F, Ma SH, Ma K, Liu H, Yan Z, Liu J, Abrigo J, Soo YOY, Liebeskind DS, Wong KS, Leung TW (2018) Translesional pressure gradient and leptomeningeal collateral status in symptomatic middle cerebral artery stenosis. Eur J Neurol 25: 404-410. https://doi.org/10.1111/ene.13521

12. Brozici M, van der Zwan A, Hillen B (2003) Anatomy and functionality of leptomeningeal anastomoses: a review. Stroke 34: 2750-2762. https://doi.org/10.1161/01.STR.0000095791.85737. 65

13. Michel E, Liu H, Remley KB, Martin AJ, Madison MT, Kucharczyk J, Truwit CL (2001) Perfusion MR neuroimaging in patients undergoing balloon test occlusion of the internal carotid artery. AJNR Am J Neuroradiol 22:1590-1596

14. Kluytmans M, van der Grond J, van Everdingen KJ, Klijn CJM, Kappelle LJ, Viergever MA (1999) Cerebral hemodynamics in 
relation to patterns of collateral flow. Stroke 30:1432-1439. https:// doi.org/10.1161/01.str.30.7.1432

15. Omura-Matsuoka E, Yagita Y, Sasaki T, Terasaki Y, Oyama N, Sugiyama Y, Todo K, Sakoda S, Kitagawa K (2011) Hypertension impairs leptomeningeal collateral growth after common carotid artery occlusion: restoration by antihypertensive treatment. J Neurosci Res 89:108-116. https://doi.org/10.1002/jnr. 22522
16. Chan S-L, Sweet JG, Bishop N, Cipolla MJ (2016) Pial collateral reactivity during hypertension and aging. Stroke 47:1618-1625. https://doi.org/10.1161/STROKEAHA.116.013392

Publisher's note Springer Nature remains neutral with regard to jurisdictional claims in published maps and institutional affiliations. 